\title{
ARTICLE
}

Lymphoma

\section{Progression-free survival at 24 months and subsequent survival of patients with extranodal NK/T-cell lymphoma: a China Lymphoma Collaborative Group (CLCG) study}

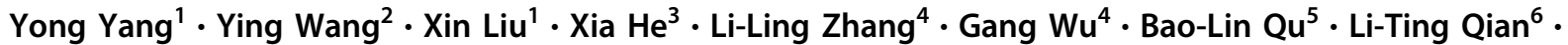

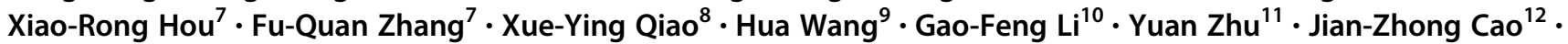

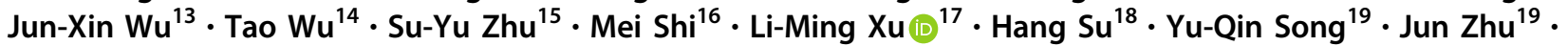 \\ Yu-Jing Zhang ${ }^{20} \cdot$ Hui-Qiang Huang ${ }^{20} \cdot$ Chen Hu ${ }^{21} \cdot$ Shu-Nan Qi ${ }^{1}{ }^{1} \cdot$ Ye-Xiong Li ${ }^{1}{ }^{1}$
}

Received: 3 June 2020 / Revised: 11 August 2020 / Accepted: 7 September 2020 / Published online: 17 September 2020

(c) The Author(s) 2020. This article is published with open access

\begin{abstract}
Limited evidence supports the use of early endpoints to evaluate the success of initial treatment of extranodal NK/T-cell lymphoma (ENKTCL) in the modern era. We aim to analyze progression-free survival at 24 months (PFS24) and subsequent overall survival (OS) in a large-scale multicenter cohort of patients. 1790 patients were included from the China Lymphoma Collaborative Group (CLCG) database. Subsequent OS was defined from the time of PFS24 or progression within 24 months to death. OS was compared with age- and sex-matched general Chinese population using expected survival and standardized mortality ratio (SMR). Patients who did not achieve PFS24 had a median OS of 5.3 months after progression, with 5-year OS rate of 19.2\% and the SMR of 71.4 (95\% CI, 62.9-81.1). In contrast, 74\% patients achieved PFS24, and the SMR after achieving PFS24 was 1.77 (95\% CI, 1.34-2.34). The observed OS rate after PFS24 versus expected OS rate at 5 years was $92.2 \%$ versus $94.3 \%$. Similarly, superior outcomes following PFS24 were observed in early-stage patients (5-year OS rate, 92.9\%). Patients achieving PFS24 had excellent outcome, whereas patients exhibiting earlier progression had a poor survival. These marked differences suggest that PFS24 may be used for study design and risk stratification in ENKTCL.
\end{abstract}

\section{Introduction}

Extranodal NK/T-cell lymphoma, nasal type (ENKTCL) is an aggressive and heterogeneous disease with an unusual geographical distribution. Although rare globally, it is more prevalent in East Asia and South America [1-4]. Clinically, the majority of patients with ENKTCL present with earlystage disease and are male adults. Extensive primary tumor invasion (PTI) is usually present, involving the upper aerodigestive tract (UADT) site, and extranodal failure is common [5-7]. Over the past decade, substantial treatment advances

These authors contributed equally: Yang Y, Wang Y, Liu X

Shu-Nan Qi

medata@163.com

Ye-Xiong Li

yexiong12@163.com

Extended author information available on the last page of the article have been made with the introduction of novel prognostic models and risk stratifications [8-10], upfront radiotherapy [11-14], and non-anthracycline (ANT)-based chemotherapy [15-21]. The treatment outcome has improved, with 5-year overall survival (OS) rates of 60-90\% reported for localized diseases, but only $10-40 \%$ for disseminated diseases [10, 13, 22-25]. A large proportion of patients suffers disease progression or relapse after employment of the current standard treatment. Thus, there is an urgent requirement for the identification of early efficacy endpoints or novel therapeutic agents from prospective trials in patients with ENKTCL.

Previous studies have demonstrated that event-free survival (EFS) at 24 months (EFS24) and progression-free survival (PFS) at 24 months (PFS24) are important milestones to stratify patients with diffuse large B-cell lymphoma (DLBCL) and peripheral T-cell lymphoma (PTCL); survival beyond these time points is similar or equivalent to that of the general population [26-29]. Normalization to the survival of a country-matched general population was recently proposed as an alternative to the use of "time to event" analysis for lymphoma. However, clinical 
characteristics and treatment strategies differ notably between lymphoma subtypes. The clinical significance of PFS24 in ENKTCL remains unknown, and the impact of achieving PFS24 on subsequent OS remains unaddressed. In the present study, we aimed to examine the timing of events, post-treatment milestones and subsequent OS in patients with ENKTCL, with reference to the general background population.

\section{Patients and methods}

\section{Eligibility criteria and study population}

Patients diagnosed with ENKTCL between 2008 and 2016 were retrospectively reviewed using information from the China Lymphoma Collaborative Group (CLCG) database $[5,9,13,21]$. The eligibility criteria in this study included patients who had received non-ANT-based chemotherapy and/or radiotherapy. Patients who had received ANT-based or unknown regimen chemotherapy were excluded. A total of 1790 patients formed the study population. The institutional review boards ethically approved this project and waived the requirement for informed consent because of the de-identification of patient data.

\section{Risk stratification and treatment}

Patients were staged using the Ann Arbor staging system and stratified using the ENKTCL-specific models: the nomogram-revised risk index [8,9], the prognostic index of natural killer lymphoma [10] and the Korea prognostic index [30]. Patients with early-stage disease received combined modality treatment $(n=1091,69.8 \%)$, radiotherapy alone $(n=283,18.1 \%)$ or chemotherapy alone $(n=189,12.1 \%)$. Patients with advanced-stage disease received primary chemotherapy with $(n=89,39.2 \%)$ or without $(n=138,60.8 \%)$ consolidation radiotherapy. The most commonly used non-ANT-based chemotherapies were asparaginase- or platinum-containing $(n=1367$, 90.7\%). Extended involved-site radiation therapy (E-ISRT) was administered, with a median dose of $50 \mathrm{~Gy}$.

\section{Statistical methods}

Progression-free survival (PFS) was defined as the time from the date of treatment to the first of either disease progression, relapse or death from any cause. Subsequent OS was defined as the time from achieving PFS24 (24 months after treatment) or time from progression in patients who did not achieve PFS24 (progression within 24 months of treatment) to death from any cause.
The estimated hazard rates provide the trajectory of progression and death overtime and were smoothed by the Epanechnikov kernel. PFS24 was defined as being alive and progression-free 24 months after initial therapy. PFS was also evaluated at other landmark time points (12 and 36 months) for sensitivity analysis. OS was compared with the age- and sex-matched general Chinese population via standardized mortality ratios (SMRs), and expected survival was predicted using a conditional approach via the survexp' function in $\mathrm{R}$ (package survival). Survival curves we analyzed using Kaplan-Meier method. A two-sided $P$ value of $<0.05$ was considered to indicate a statistically significant difference. Statistical analyses were performed using SPSS (version 22.0; IBM Inc.) and R (version 3.6.2; http://www.r-project.org/).

\section{Results}

\section{Patient characteristics and survival}

The baseline clinical characteristics of the included patients are listed in Table 1 . The median age was 44 years old (interquartile range, 32-55 years) and the male:female ratio was 2.4:1. Most patients had good performance status and primary UADT site. Elevated lactate dehydrogenase was noted in $27.6 \%$ of patients, and the majority exhibited earlystage disease $(87.3 \%)$.

With a median follow-up time of 46 months for surviving patients, 598 patients $(33.4 \%)$ exhibited disease progression and 430 patients $(24.0 \%)$ passed away. The 5-year OS and PFS rates for all patients were $72.5 \%$ and $62.2 \%$, respectively.

\section{Annual hazard rate over time}

After initial treatment, $82 \%$ of progression and $80 \%$ of mortalities occurred within 24 months. Consistently, smoothed hazard plots illustrated maximal annual death and progression hazards of $15.4 \%$ and $27.1 \%$, respectively during the first year. These hazard rates decreased to $6.1 \%$ and $7.6 \%$, respectively, at 2 years (Fig. 1a). From 3 years the annual hazards for progression and death were $<5 \%$. Thus, PFS at 24 months (PFS24) is a logistical cutoff time point for further evaluation.

\section{PFS24 and subsequent OS}

Kaplan-Meier analysis estimated that the 2-year PFS rate of the total cohort (1790 patients) was $70.5 \%$ (95\% CI, 68.3-72.7\%) and the SMR from initial treatment was 6.7 (95\% CI, 6.1-7.3). The 5-year OS and SMR among subgroups were shown in Table 1. 
Table 1 Clinical characteristics and treatment outcomes of patients with extranodal nasal-type NK/T-cell lymphoma.

\begin{tabular}{|c|c|c|c|c|c|}
\hline \multirow[b]{2}{*}{ Characteristics } & \multirow{2}{*}{$\begin{array}{l}\text { Patients } \\
\text { No. }(\%)\end{array}$} & \multicolumn{2}{|c|}{ 5-year OS rate post-treatment } & \multicolumn{2}{|l|}{ SMR } \\
\hline & & $\%(95 \% \mathrm{CI})$ & $P$ & $(95 \% \mathrm{CI})$ & $P^{\mathrm{a}}$ \\
\hline Sex & & & 0.181 & & \\
\hline Male & $1261(70.4)$ & $71.7(68.9-74.6)$ & & $5.6(5.1-6.3)$ & $<0.001$ \\
\hline Female & $529(29.6)$ & $74.5(70.4-78.7)$ & & $1.3(1.1-1.5)$ & $<0.001$ \\
\hline Age (years) & & & 0.013 & & \\
\hline$\leq 60$ & $1527(85.3)$ & $73.5(71.1-76.0)$ & & $14.7(13.2-16.3)$ & $<0.001$ \\
\hline$>60$ & $263(14.7)$ & $66.4(59.9-73.6)$ & & $1.9(1.5-2.3)$ & $<0.001$ \\
\hline Primary site & & & $<0.001$ & & \\
\hline UADT & $716(40.0)$ & $73.9(71.6-76.3)$ & & $6.3(5.7-7.0)$ & $<0.001$ \\
\hline Extra-UADT & $1074(60.0)$ & $45.4(34.0-60.8)$ & & $14.3(10.6-19.3)$ & $<0.001$ \\
\hline Regional lymph nodes & & & $<0.001$ & & \\
\hline Yes & $1674(93.5)$ & $64.7(60.9-68.8)$ & & $13.3(11.6-15.2)$ & $<0.001$ \\
\hline No & $116(6.5)$ & $77.4(74.6-80.3)$ & & $4.5(3.9-5.1)$ & $<0.001$ \\
\hline Distant lymph nodes & & & $<0.001$ & & \\
\hline Yes & $1671(93.4)$ & $46.3(35.7-60.2)$ & & $17.8(13.2-24.2)$ & $<0.001$ \\
\hline No & $119(5.6)$ & $73.8(71.5-76.2)$ & & $6.2(5.7-6.9)$ & $<0.001$ \\
\hline Primary tumor invasion & & & $<0.001$ & & \\
\hline Yes & $1004(56.1)$ & $67.9(64.8-71.2)$ & & $9.7(8.6-10.8)$ & $<0.001$ \\
\hline No & $786(43.9)$ & $78.4(75.1-81.8)$ & & $4.1(3.5-4.8)$ & $<0.001$ \\
\hline B symptoms & & & 0.006 & & \\
\hline Yes & $687(38.4)$ & $68.9(65.2-72.9)$ & & $9.9(8.6-11.5)$ & $<0.001$ \\
\hline No & 1103 (61.6) & $74.8(71.9-77.8)$ & & $5.3(4.6-6.0)$ & $<0.001$ \\
\hline Elevated LDH & & & $<0.001$ & & \\
\hline Yes & $100(5.6)$ & $60.1(55.3-65.3)$ & & $1.2(1.1-1.4)$ & $<0.001$ \\
\hline No & $1690(94.4)$ & $77.1(74.5-79.7)$ & & $5.1(4.5-5.8)$ & $<0.001$ \\
\hline ECOG score & & & $<0.001$ & & \\
\hline $0-1$ & $1563(87.3)$ & $74.7(72.4-77.1)$ & & $6.2(5.6-6.8)$ & $<0.001$ \\
\hline$\geq 2$ & $227(12.7)$ & $39.1(30.2-50.7)$ & & $12.9(10.1-16.5)$ & $<0.001$ \\
\hline Ann Arbor stage & & & $<0.001$ & & \\
\hline I-II & $1296(72.4)$ & $75.9(73.5-78.3)$ & & $5.5(5.0-6.1)$ & $<0.001$ \\
\hline III-IV & 494 (27.6) & $44.4(36.7-53.7)$ & & $24.4(19.9-29.8)$ & $<0.001$ \\
\hline KPI & & & $<0.001$ & & \\
\hline Group 1 & $550(30.7)$ & $81.8(78.3-85.5)$ & & $3.1(2.5-3.8)$ & $<0.001$ \\
\hline Group 2 & $636(35.5)$ & $75.0(71.2-79.0)$ & & $6.2(5.2-7.3)$ & $<0.001$ \\
\hline Group 3 & $373(20.9)$ & $67.4(62.4-72.9)$ & & $10.6(8.7-12.8)$ & $<0.001$ \\
\hline Group 4 & $231(12.9)$ & $49.6(42.6-57.9)$ & & $27.9(22.9-34.1)$ & $<0.001$ \\
\hline PINK & & & $<0.001$ & & \\
\hline Low risk & $1269(70.9)$ & $78.1(75.6-80.6)$ & & $11.4(10.1-12.9)$ & $<0.001$ \\
\hline Intermediate risk & $363(20.3)$ & $61.7(55.9-68.1)$ & & $3.1(2.6-3.7)$ & $<0.001$ \\
\hline High risk & $158(8.8)$ & $45.5(36.6-56.6)$ & & $12.8(10.0-16.3)$ & $<0.001$ \\
\hline NRI & & & $<0.001$ & & \\
\hline Low risk & $390(21.8)$ & $86.4(82.6-90.3)$ & & $5.6(4.2-7.4)$ & $<0.001$ \\
\hline Intermediate-low risk & $517(28.9)$ & $78.3(74.3-82.5)$ & & $3.9(3.2-4.8)$ & $<0.001$ \\
\hline Intermediate-high risk & $469(26.2)$ & $69.8(65.3-74.8)$ & & $7.0(5.9-8.4)$ & $<0.001$ \\
\hline High risk & $263(14.7)$ & $59.4(52.9-66.6)$ & & $11.0(8.9-13.6)$ & $<0.001$ \\
\hline Very high risk & $151(8.4)$ & $43.2(24.8-53.7)$ & & $12.2(9.7-15.3)$ & $<0.001$ \\
\hline
\end{tabular}

$O S$ overall survival, $S M R$ standardized mortality ratio, $C I$ confidence interval, $U A D T$ upper aerodigestive tract, $L D H$ lactate dehydrogenase, ECOG Eastern Cooperative Oncology Group, KPI Korean Prognostic Index, PINK Prognostic Index of Natural Killer Lymphoma, NRI nomogramrevised risk index.

${ }^{a}$ Compared with the age- and sex-matched general Chinese population.

A total of 1362 patients were sufficiently followed-up for PFS24 assessment. Of these patients, 1014 (74.4\%) were progression-free at 24 months (PFS24 was achieved). The 5-year OS rate after achieving PFS24 was $92.2 \%$ (95\% CI,
89.6-94.9\%; Fig. 1b). The expected 5-year OS rate was $94.3 \%$ in the age and sex-matched general Chinese population. The SMR after achieving PFS24 was 1.8 (95\% CI, $1.3-2.3 ; \quad P<0.001)$. In contrast, the median OS after 

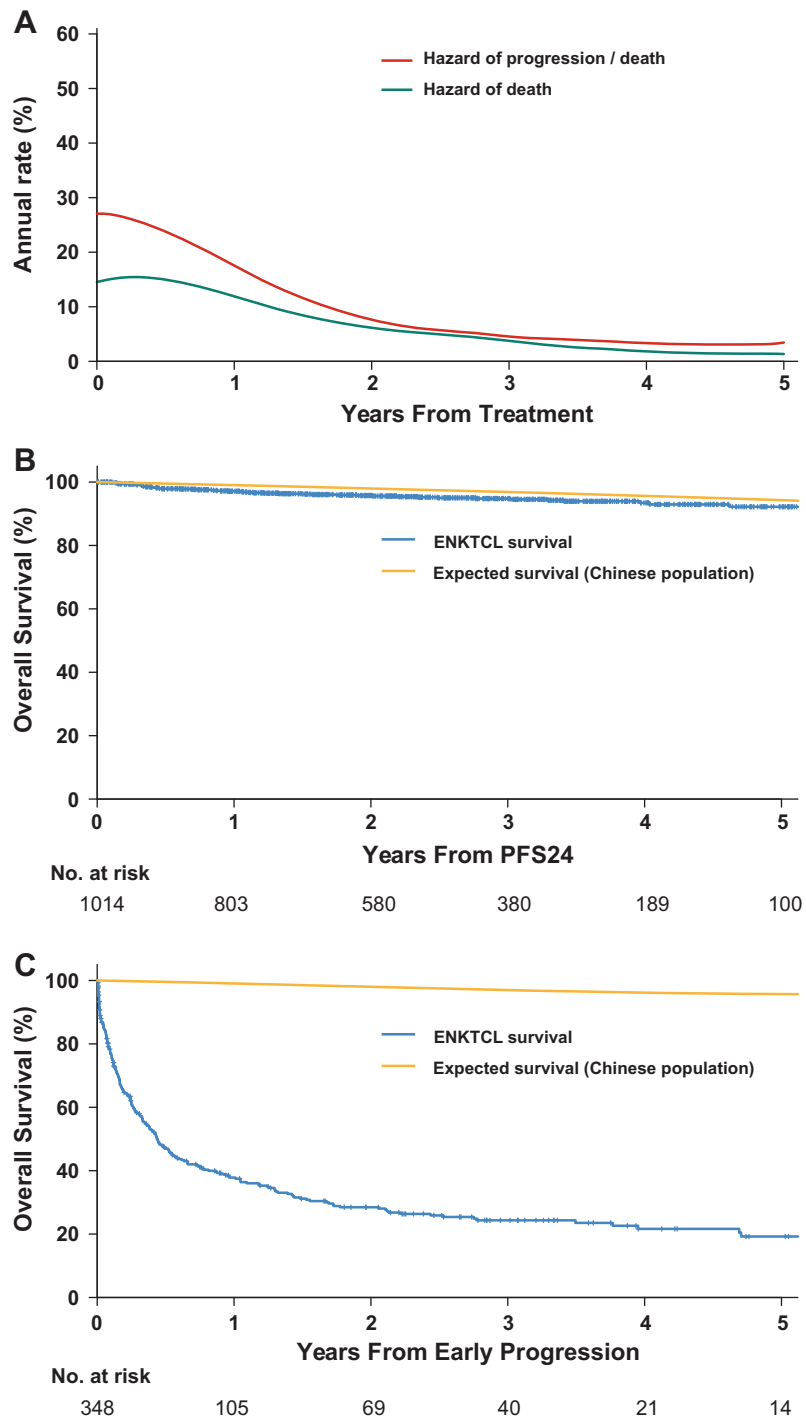

Fig. 1 Estimated annual hazards and overall survival (OS) based on 24-month progression-free survival (PFS24) in the whole cohort. a Smoothed hazard plots of death and progression over time. b Subsequent OS of patients who achieved PFS24 after initial treatment vs. expected OS based on age-, sex-, and country-matched general population data. c Subsequent OS of patients who did not achieve PFS24 after initial treatment vs. expected OS based on age-, sex-, and country-matched general population data.

progression within the first 24 months was only 5.3 months (95\% CI, 4.0-6.7). The 5-year OS rate after progression was 19.2\% (Fig. 1c) and SMR was 71.4 (95\% CI, 62.9-81.0; $P<0.001)$.

\section{Subgroup analysis of PFS24 and subsequent OS}

In early-stage disease, 950/1239 (76.7\%) patients achieved PFS24 and 289/1239 (23.3\%) patients did not. The 5-year OS rate after achieving PFS24 was $92.9 \%$ (95\% CI, 90.2\% to $95.6 \%$ ), almost equivalent to that of the age and sexmatched general population $(94.3 \%$, Fig. 2a). The SMR after achieving PFS24 was $1.5 \quad$ (95\% CI, 1.1-2.0; $P=0.009$ ). In contrast, the median OS after progression within the first 24 months was only 5.0 months (95\% CI, 3.7-6.3). The 5-year OS rate after progression was $20.1 \%$ (Fig. 2b) and SMR was 67.3 (95\% CI, 58.5- 77.2; $P<0.001)$.

In advanced-stage disease, 123 patients were sufficiently followed-up for PFS24 assessment. PFS24 was achieved for $52.0 \%$ patients. The 3-year OS rate after achieving PFS24 was $83.1 \%$ (95\% CI, 73.4-94.2\%), which is lower than that of the age and sex-matched population (97.5\%; Fig. 2c). The SMR after achieving PFS24 was 9.6 (95\% CI, 5.0-18.4; $P<0.001)$. In contrast, the median OS after progression within the first 24 months was only 6.9 months (95\% CI, 4.9-9.1 months), with a 3-year OS rate of $13.7 \%$ (Fig. 2d) and SMR of 103.6 (95\% CI, 76.0-141.3; $P<0.001)$.

Subsequently, outcomes were examined with regard to other clinical factors and risk groups. Despite a significant difference in OS of patients between treatment subgroups (Table 1), the OS rate of patients after achieving PFS24 were favorable compared with the background population (Table 2). There was little difference in subsequent outcomes after progression regardless of clinical characteristics or risk-stratified grouping (Table 2). PFS24 status was informative for subsequent $\mathrm{OS}$ in all patient subgroups and could be used as a prognostic indicator.

\section{Sensitivity analysis of PFS24 vs. other time points}

For sensitivity analysis, we examined outcomes according to other landmark time points of PFS, including 12 (PFS12), 24 (PFS24) and 36 (PFS36) months. Although the subsequent 5-year OS rate continued to increase from PFS12 (86.5\%) to PFS24 (92.2\%), there was little benefit in examining beyond PFS36 (93.0\%) and no significant difference between the PFS-based OS and expected OS (Table 3). Furthermore, there was little difference in the 5 -year OS rate after progression regardless of the time point chosen (17.1\% at 12 months vs. $20.3 \%$ at 36 months). Similar results were obtained when considering early-stage and advanced-stage diseases.

\section{Discussion}

This is the first study to evaluate PFS-based endpoints among patients with ENKTCL, primarily treated with nonANT-based chemotherapy and frontline radiotherapy. Using a large multicenter cohort of patient data collected from the CLCG database, we verified that PFS24 acts as a prognostic indicator of subsequent survival under current treatment strategies. Patients who were progression-free 
A

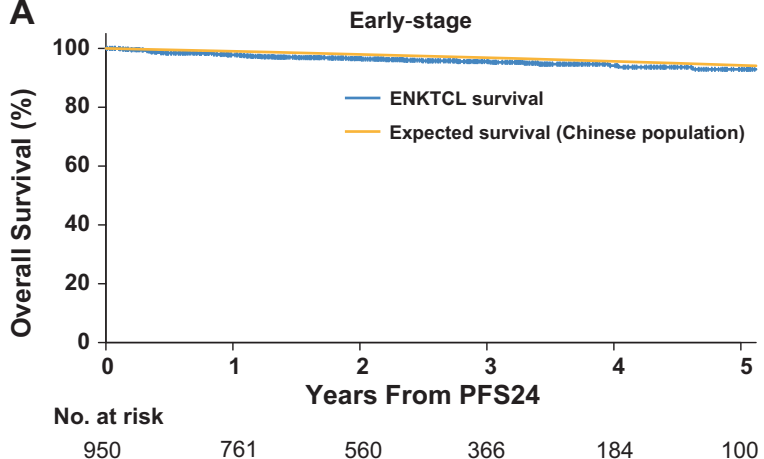

C

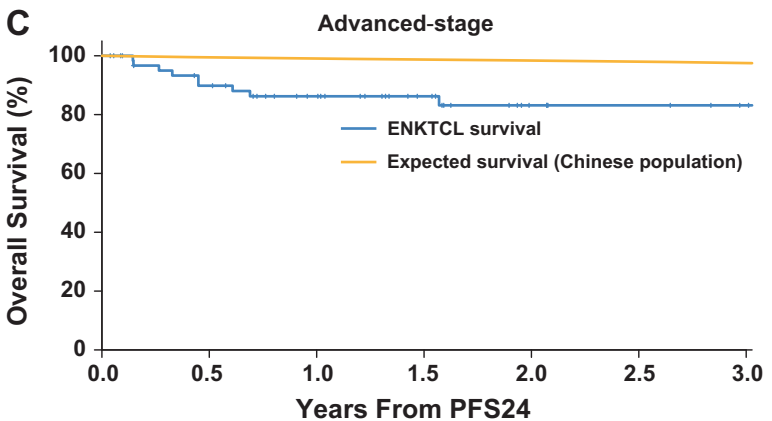

No. at risk

$\begin{array}{lllllll}62 & 54 & 42 & 31 & 20 & 17 & 14\end{array}$

Fig. 2 Overall survival (OS) based on 24-month progression-free survival (PFS24) of patients with early-stage and advanced-stage. a Subsequent OS of patients who achieved PFS24 after initial treatment vs. expected OS based on age-, sex- and country-matched general population data. b Subsequent OS of early-stage patients who did not achieve PFS24 vs. expected OS based on age-, sex-, and

within 24 months after initial treatment resulted in favorable long-term outcomes, with OS times not distinguishable from that of age-, sex- and country-matched populations. Conversely, patients who experienced disease progression within 24 months had poor prognoses. This finding suggests PFS24 as a dichotomous variable, providing a clear benchmark for evaluating the success of initial treatment and for designing clinical trials for ENKTCL.

The introduction of asparaginase- or platinum-based chemotherapy regimens and early implementation of highdose E-ISRT for localized disease have been the most important advances in the treatment of ENKTCL [11-21, 31]. Although phase I/II trials or retrospective studies have reported improved survival with the use of non-ANT-based chemotherapy, the optimal chemotherapy regimens remain to be identified by prospective trials. Early efficacy endpoints such as PFS and EFS are required to reduce the duration of evaluation of treatment regimens, to deliver optimal treatment strategies to the clinic sooner, and so that ineffective regimens can be abandoned without prolonged evaluation [32]. However, due to the rarity of ENKTCL and the heterogeneity of treatments used, limited patient numbers make formal surrogate endpoint analysis

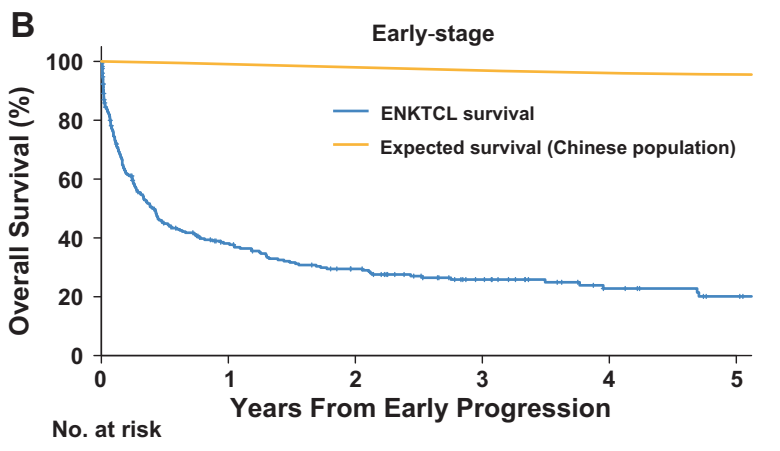

No. at risk

90

$63 \quad 37$

20

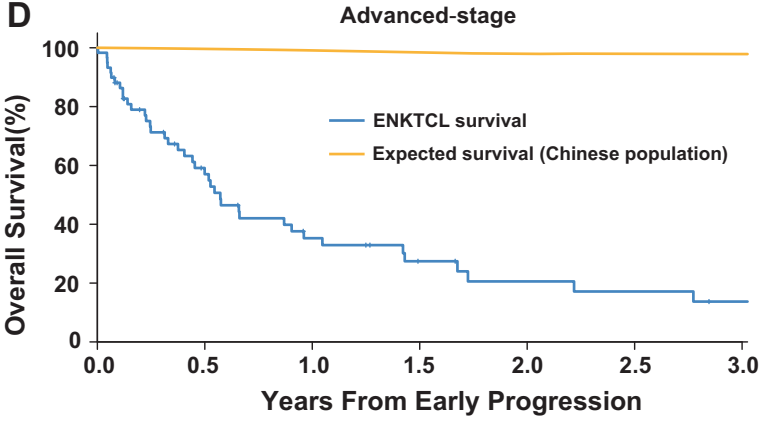

No. at risk

$\begin{array}{lllllll}59 & 27 & 15 & 9 & 7 & 6 & 4\end{array}$

country-matched general population data. c Subsequent OS of advanced-stage patients who achieved PFS24 vs. expected OS based on age-, sex-, and country-matched general population data. d Subsequent OS of advanced-stage patients who did not achieve PFS24 vs. expected OS based on age-, sex-, and country-matched general population data.

difficult. Normalization to the survival of a matched general population is important in rare diseases such as PTCL and ENKTCL [29]. In the present study, PFS24 is identified as a dichotomous variable that may allow individualized prognosis prediction for patients with ENKTCL, as well as inform patient counseling, clinical decision making and prospective clinical trial design.

In previous studies, we have demonstrated that improved locoregional control is associated with prolonged PFS and OS in early-stage ENKTCL [33], and that the survival probability increased and the hazards of failure decreased in a risk-dependent manner [34]. In the present study, we demonstrate that the estimated risk of disease progression and mortality was initially high, but decreased dramatically within the first 24 months after current standard treatment for ENKTCL (Fig. 1a). Given that the vast majority of ENKTCL-related events occur within the first 24 months, patients achieving PFS24 are highly likely to have a nearnormal life expectancy, with actual and expected 5-year OS rates of $92.2 \%$ and $94.3 \%$. Sensitivity analysis, examining time points at 12,24 , and 36 months, indicated that there was little OS improvement at PFS36, whereas the PFS12 endpoint did not dichotomize the two groups as effectively 


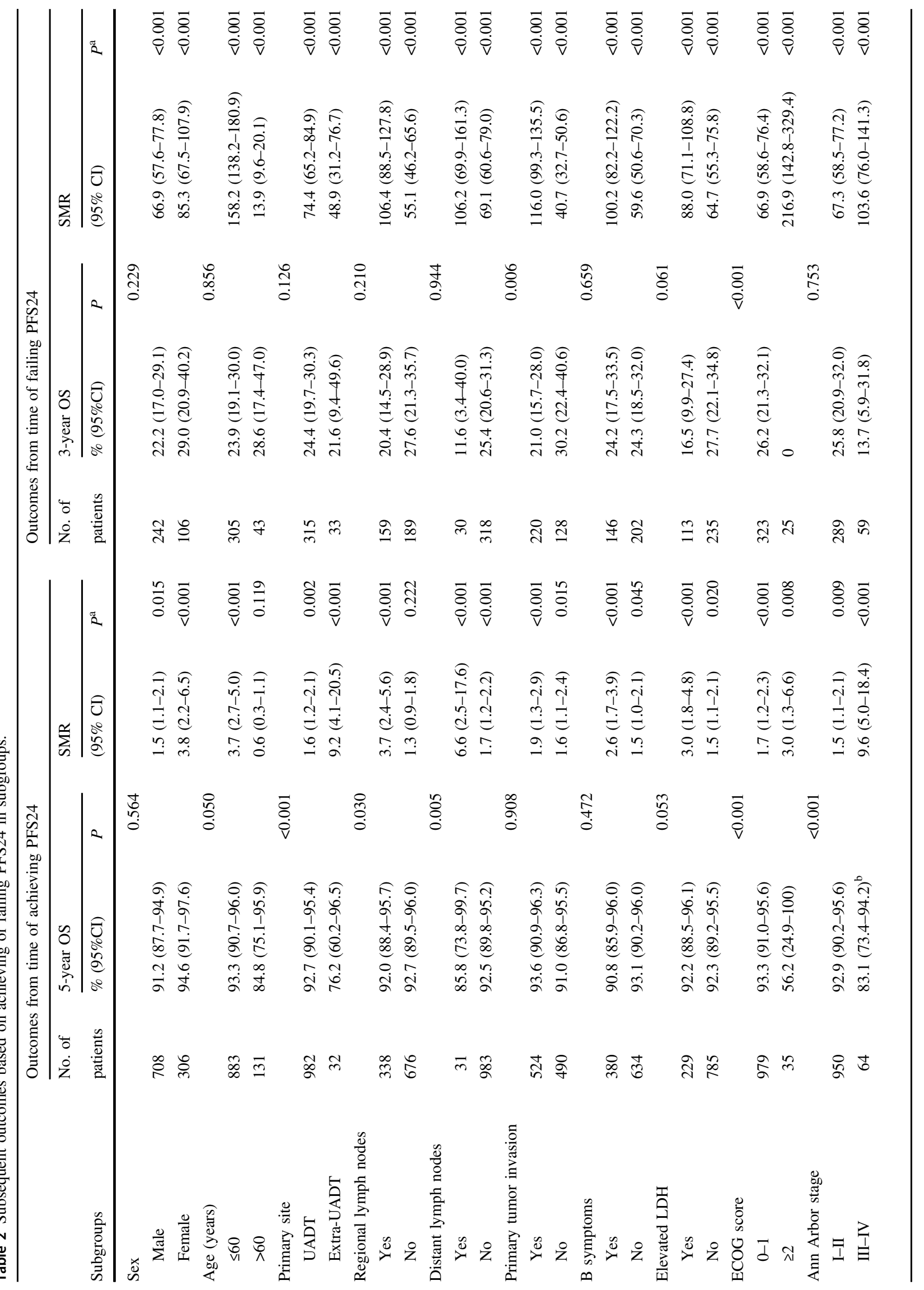




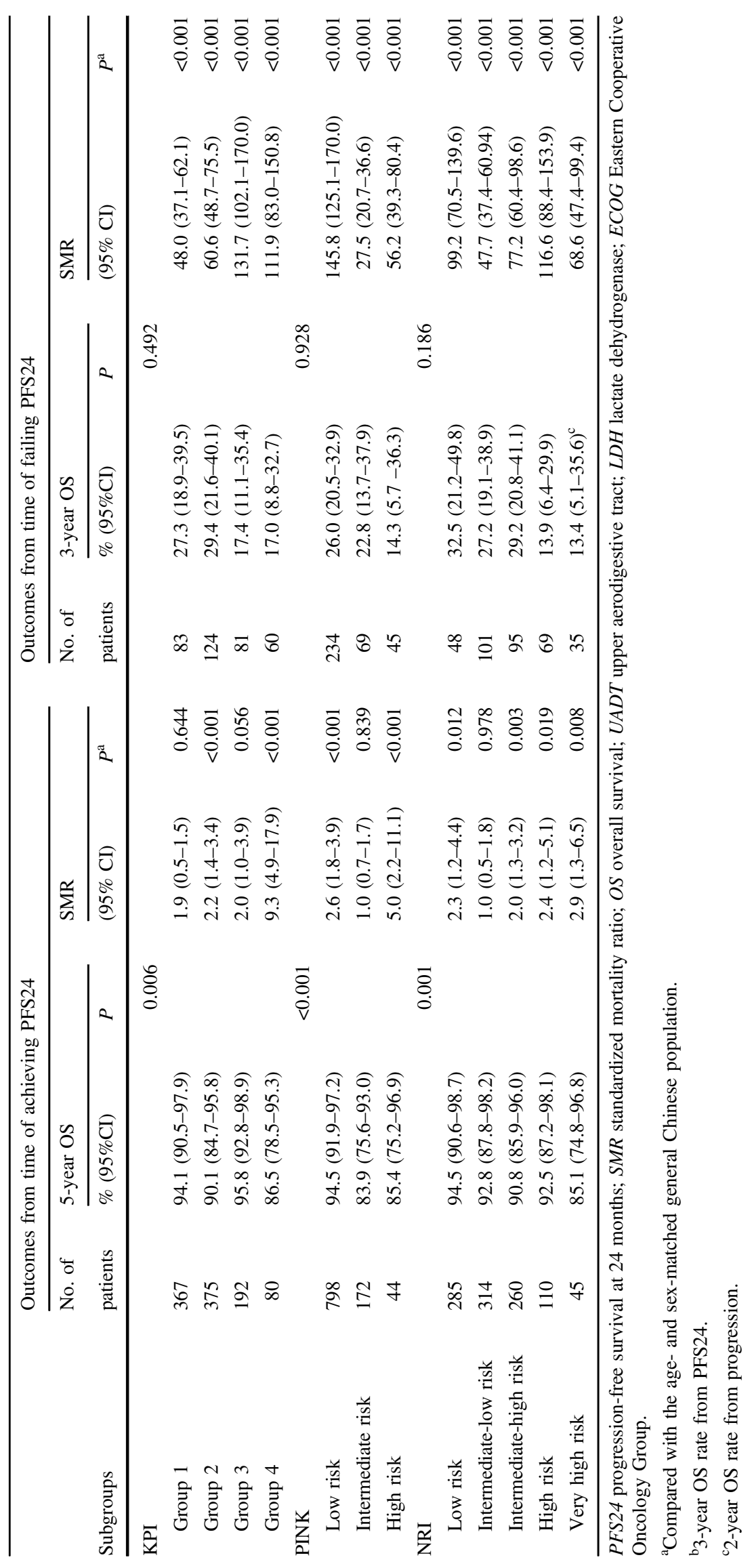




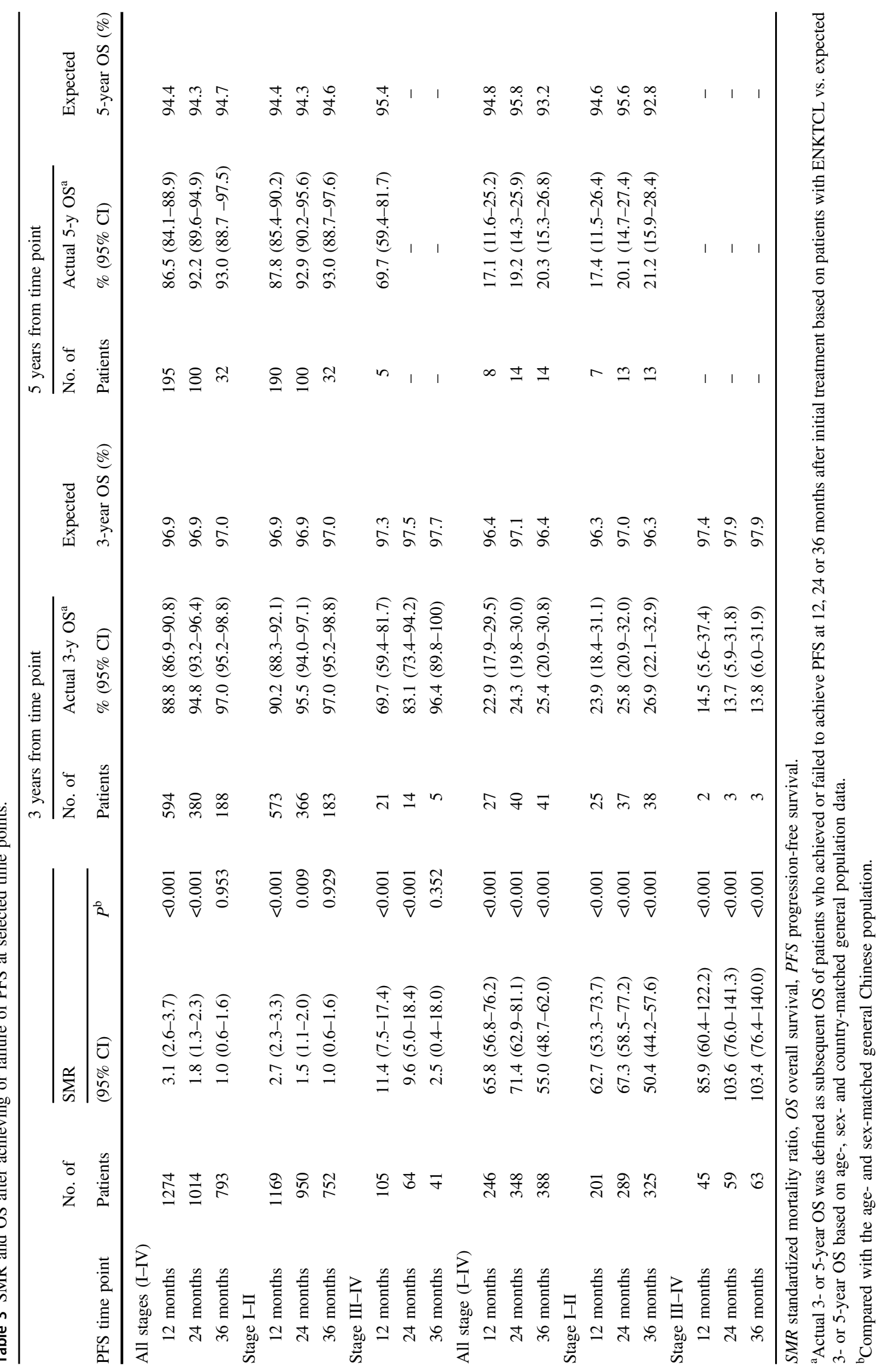


as PFS24, regardless of stage. In consistence with the observations of the current study, other studies have reported PFS24 to be an important endpoint for DLBCL and PTCL, associated with subsequent long-term outcomes [28, 29]. Regarding ENKTCL patients who failed to achieve PFS24, the median OS after progression was only 5.3 months, indicating that few patients who suffer early progression can be successfully treated. As observed by other studies [35, 36], ENKTCL patients who suffer disease progression after initial treatment had extremely poor prognoses, regardless of stage or risk group. In a recent study of 179 patients with ENKTCL who suffered disease relapse or progression after initial non-ANT-based chemotherapy, the median OS was only 6.4 months [35]. Similarly, for patients who failed to achieve PFS24 or EFS24, the subsequent median OS was 7.2 months after ANT-based immunochemotherapy for DLBCL and 4.9 months after ANT-based chemotherapy for PTCL $[28,29]$. These findings indicate that PFS24 could be safely incorporated into routine clinical practice without detrimental effects on long-term disease control or survival outcomes in aggressive lymphomas under current standard treatments. PFS24 should be further validated in the context of randomized controlled trials as a potential early efficacy endpoint for patients with ENKTCL. Furthermore, there is an urgent requirement to identify patients at high risk of early progression who would benefit from innovative treatment strategies. Following the achievement of PFS24, research for ENKTCL survivors should focus on lessintensive surveillance, long-term treatment toxicity, quality of life, and other outcomes [34, 37, 38].

Previous studies, mainly using the randomized controlled trial (RCT) or real-world data, have demonstrated that the surrogate endpoints of EFS and PFS are strongly related to OS at both trial- and individual-level in different lymphoma subtypes [26-29, 32, 39-41]. Consistent with the present finding, the subsequent survival of patients with DLBCL or PTCL who achieved EFS24 or PFS24 is almost equal to that of the age-, sex-, and country-matched general population [26-29, 32]. Furthermore, we have confirmed the association of PFS24 with OS in trial- and treatment armlevel in RCTs on DLBCL patients treated with immunochemotherapy; its association has been externally validated using the literature-based data from high-quality phase II and retrospective studies [40]. Similarly, in patients with follicular lymphoma and marginal zone lymphomas [39, 41], early progression of disease within 24 months after initial treatment stratified subsequent OS and identifies a high-risk population. Consistent with the present study, these findings highlight the important role of PFS or EFS as early efficacy endpoint in designing prospective trials. The further work is needed to determine the prognostic factors for PFS24 in patients with ENKTCL.
The limitations of this study are as follows: first, the impact of salvage treatment on survival outcome after progression was not assessed, as it is beyond the scope of this study. Originally, our dataset was designed to collect only baseline characteristics, initial treatments, and events during follow-up. Second, these findings were mostly based on patients with early-stage disease, primarily treated with non-ANT-based regimens and radiotherapy. Extrapolation of these results when considering patients who underwent other treatments would be speculative. Third, despite the wide enrollment of ENKTCL patients primarily treated with non-ANT-based chemotherapy and upfront radiotherapy from a real-world CLCG database, more work is required to externally validate the correlation between PFS24 and OS using independent data from other large database, particularly from nonendemic areas. Furthermore, the retrospective design of this study does not allow for assessment of EFS as endpoint. The evaluation of EFS24 vs. PFS24 may be explored in a future study using CLCG data. The patients included in this study represent a young population with few comorbidities; longer follow-up and comparison to other collaboration data will give more insight into the importance of these endpoints.

In conclusion, assessment of PFS24 stratifies subsequent outcomes in patients with ENKTCL. Patients who achieve PFS24 have favorable subsequent survival regardless of stage and risk group. However, patients who suffer early progression have extremely poor prognoses. These results support the use of PFS24 as an efficacy endpoint, and should be considered in future studies evaluating novel therapies and biomarkers for risk stratification.

Acknowledgements This work was supported by grants from the National Natural Science Foundation of China (81670185), Chinese Academy of Medical Science (CAMS), Innovation Fund for Medical Sciences (CIFMS, 2016-I2M-1-001), and the National Key Projects of Research and Development of China (2016YFC0904600). The funding sources had no influence on the design, performance, or reporting of this study.

Author contributions Conception and design: Y-XL, S-NQ, YY, XL. Financial support: Y-XL, YW. Administrative support: Y-XL, YW. Provision of study material or patients: YY, XL, YW, XH, L-LZ, GW, B-LQ, L-TQ, X-RH, X-YQ, HW, G-FLi, YZ, J-ZC, J-XW, TW, S-YZ, MS, L-MX, HS, Y-QS, Y-JZ, S-NQ, and Y-XL. Collection and assembly of data: YY, XL. Data analysis and interpretation: YY, XL, YW, S-NQ, CH, Y-XL. Manuscript writing: all authors. Final approval of manuscript: all authors. Accountable for all aspects of the work: all authors.

\section{Compliance with ethical standards}

Conflict of interest $\mathrm{CH}$ has received grants from National Cancer Institute and personal fees from Merck \& Co., outside the submitted work. All other authors declare no competing interests.

Publisher's note Springer Nature remains neutral with regard to jurisdictional claims in published maps and institutional affiliations. 
Open Access This article is licensed under a Creative Commons Attribution 4.0 International License, which permits use, sharing, adaptation, distribution and reproduction in any medium or format, as long as you give appropriate credit to the original author(s) and the source, provide a link to the Creative Commons license, and indicate if changes were made. The images or other third party material in this article are included in the article's Creative Commons license, unless indicated otherwise in a credit line to the material. If material is not included in the article's Creative Commons license and your intended use is not permitted by statutory regulation or exceeds the permitted use, you will need to obtain permission directly from the copyright holder. To view a copy of this license, visit http://creativecommons. org/licenses/by/4.0/.

\section{References}

1. Yang QP, Zhang WY, Yu JB, Zhao S, Xu H, Wang W, et al. Subtype distribution of lymphomas in Southwest China: analysis of 6382 cases using WHO classification in a single institution. Diagn Pathol. 2011;6:77.

2. Sun J, Yang Q, Lu Z, He M, Gao L, Zhu M, et al. Distribution of lymphoid neoplasms in China: analysis of 4638 cases according to the World Health Organization classification. Am J Clin Pathol. 2012;138:429-34.

3. Au WY, Weisenburger DD, Intragumtornchai $T$, Nakamura $S$, Kim WS, Sng I, et al. Clinical differences between nasal and extranasal natural killer/T-cell lymphoma: a study of 136 cases from the International Peripheral T-Cell Lymphoma Project. Blood. 2009;113:3931-37.

4. Adams SV, Newcomb PA, Shustov AR. Racial patterns of peripheral T-cell lymphoma incidence and survival in the United States. J Clin Oncol. 2016;34:963-71.

5. Qi SN, Xu LM, Yuan ZY, Wu T, Zhu SY, Shi M, et al. Effect of primary tumor invasion on treatment and survival in extranodal nasal-type NK/T-cell lymphoma in the modern chemotherapy era: a multicenter study from the China Lymphoma Collaborative Group (CLCG). Leuk Lymphoma. 2019;60:2669-78.

6. Li YX, Liu QF, Fang H, Qi SN, Wang H, Wang WH, et al. Variable clinical presentations of nasal and Waldeyer ring natural killer/T-cell lymphoma. Clin Cancer Res. 2009;15:2905-12.

7. Liu QF, Wang WH, Wang SL, Liu YP, Huang WT, Lu N, et al. Immunophenotypic and clinical differences between the nasal and extranasal subtypes of upper aerodigestive tract natural killer/Tcell lymphoma. Int J Radiat Oncol Biol Phys. 2014;88:806-13.

8. Yang Y, Zhang YJ, Zhu Y, Cao JZ, Yuan ZY, Xu LM, et al. Prognostic nomogram for overall survival in previously untreated patients with extranodal NK/T-cell lymphoma, nasal-type: a multicenter study. Leukemia. 2015;29:1571-77.

9. Chen SY, Yang Y, Qi SN, Wang Y, Hu C, He X, et al. Validation of nomogram-revised risk index and comparison with other models for extranodal nasal-type NK/T-cell lymphoma in the modern chemotherapy era: Indication for prognostication and clinical decision-making. Leukemia. 2020; https://doi.org/10.1038/s41375-020-0791-3.

10. Kim SJ, Yoon DH, Jaccard A, Chng WJ, Lim ST, Hong H, et al. A prognostic index for natural killer cell lymphoma after nonanthracycline-based treatment: a multicentre, retrospective analysis. Lancet Oncol. 2016;17:389-400.

11. Li YX, Yao B, Jin J, Wang WH, Liu YP, Song YW, et al. Radiotherapy as primary treatment for stage IE and IIE nasal natural killer/T-cell lymphoma. J Clin Oncol. 2006;24:181-89.

12. Li YX, Liu QF, Wang WH, Jin J, Song YW, Wang SL, et al. Failure patterns and clinical implications in early stage nasal natural killer/T-cell lymphoma treated with primary radiotherapy. Cancer. 2011;117:5203-11.

13. Yang Y, Zhu Y, Cao JZ, Zhang YJ, Xu LM, Yuan ZY, et al. Risk-adapted therapy for early-stage extranodal nasal-type NK/Tcell lymphoma: analysis from a multicenter study. Blood. 2015; 126:1424-32.

14. Deng XW, Wu JX, Wu T, Zhu SY, Shi M, Su H, et al. Radiotherapy is essential after complete response to asparaginasecontaining chemotherapy in early-stage extranodal nasal-type NK/ T-cell lymphoma: a multicenter study from the China Lymphoma Collaborative Group (CLCG). Radiother Oncol. 2018;129:3-9.

15. Kim SJ, Kim K, Kim BS, Kim CY, Suh C, Huh J, et al. Phase II trial of concurrent radiation and weekly cisplatin followed by VIPD chemotherapy in newly diagnosed, stage IE to IIE, nasal, extranodal NK/T-cell lymphoma: Consortium for improving survival of lymphoma study. J Clin Oncol. 2009;27:6027-32.

16. Yamaguchi M, Tobinai K, Oguchi M, Ishizuka N, Kobayashi Y, Isobe Y, et al. Phase I/II study of concurrent chemoradiotherapy for localized nasal natural killer/T-cell lymphoma: Japan Clinical Oncology Group Study JCOG0211. J Clin Oncol. 2009; 27:5594-600.

17. Wang L, Wang ZH, Chen XQ, Li YJ, Wang KF, Xia YF, et al. First-line combination of gemcitabine, oxaliplatin, and L-asparaginase (GELOX) followed by involved-field radiation therapy for patients with stage IE/IIE extranodal natural killer/Tcell lymphoma. Cancer. 2013;119:348-55.

18. Qi F, Wang WH, He XH, Chen B, Gui L, Fang H, et al. Phase 2 study of first-line intensity-modulated radiation therapy followed by gemcitabine, dexamethasone and cisplatin for high-risk earlystage extranodal nasal-type NK/T-cell lymphoma: the GREEN study. Int J Radiat Oncol Biol Phys. 2018;102:61-70.

19. Yamaguchi M, Kwong YL, Kim WS, Maeda Y, Hashimoto C, Suh C, et al. Phase II study of SMILE chemotherapy for newly diagnosed stage IV, relapsed, or refractory extranodal natural killer (NK)/T-cell lymphoma, nasal type: the NK-Cell Tumor Study Group study. J Clin Oncol. 2011;29:4410-16.

20. Kwong YL, Kim WS, Lim ST, Kim SJ, Tang T, Tse E, et al. SMILE for natural killer/T-cell lymphoma: analysis of safety and efficacy from the Asia Lymphoma Study Group. Blood. 2012; 120:2973-80.

21. Qi SN, Yang Y, Song YQ, Wang Y, He X, Hu C, et al. First-line non-anthracycline-based chemotherapy for extranodal nasal-type NK/T-cell lymphoma: A retrospective analysis from the CLCG. Blood Adv. 2020;13:3141-53.

22. Yamaguchi M, Suzuki R, Oguchi M, Asano N, Amaki J, Akiba T, et al. Treatments and outcomes of patients with extranodal natural killer/T-cell lymphoma diagnosed between 2000 and 2013: a cooperative study in Japan. J Clin Oncol. 2017;35:32-9.

23. Kwong YL, Kim SJ, Tse E, Oh SY, Kwak JY, Eom HS, et al. Sequential chemotherapy/radiotherapy was comparable with concurrent chemoradiotherapy for stage I/II NK/T-cell lymphoma. Ann Oncol. 2018;29:256-63.

24. Fox CP, Civallero M, Ko YH, Manni M, Skrypets T, Pileri S, et al. Survival outcomes of patients with extranodal natural-killer T-cell lymphoma: a prospective cohort study from the international T-cell Project. Lancet Haematol. 2020;7:e284-e94.

25. Vargo JA, Patel A, Glaser SM, Balasubramani GK, Farah RJ, Marks SM, et al. The impact of the omission or inadequate dosing of radiotherapy in extranodal natural killer T-cell lymphoma, nasal type, in the United States. Cancer. 2017;123:3176-85.

26. Maurer MJ, Ghesquieres H, Jais JP, Witzig TE, Haioun C, Thompson CA, et al. Event-free survival at 24 months is a robust end point for disease-related outcome in diffuse large B-cell lymphoma treated with immunochemotherapy. J Clin Oncol. 2014;32:1066-73. 
27. Jakobsen LH, Bogsted M, Brown PN, Arboe B, Jorgensen J, Larsen TS, et al. Minimal loss of lifetime for patients with diffuse large B-cell lymphoma in remission and event free 24 months after treatment: a Danish population-based study. J Clin Oncol. 2017;35:778-84.

28. Maurer MJ, Habermann TM, Shi Q, Schmitz N, Cunningham D, Pfreundschuh M, et al. Progression-free survival at 24 months (PFS24) and subsequent outcome for patients with diffuse large Bcell lymphoma (DLBCL) enrolled on randomized clinical trials. Ann Oncol. 2018;29:1822-27.

29. Maurer MJ, Ellin F, Srour L, Jerkeman M, Bennani NN, Connors $\mathrm{JM}$, et al. International assessment of event-free survival at 24 months and subsequent survival in peripheral T-cell lymphoma. J Clin Oncol. 2017;35:4019-26.

30. Lee J, Suh C, Park YH, et al. Extranodal natural killer T-cell lymphoma, nasal-type: a prognostic model from a retrospective multicenter study. J Clin Oncol. 2006;24:612-18.

31. Wu T, Yang Y, Zhu SY, et al. Risk-adapted survival benefit of IMRT in early-stage NKTCL: a multicenter study from the China Lymphoma Collaborative Group. Blood Adv. 2018;2:2369-77.

32. Shi Q, Schmitz N, Ou FS, Dixon JG, Cunningham D, Pfreundschuh M, et al. Progression-free survival as a surrogate end point for overall survival in first-line diffuse large B-cell lymphoma: an individual patient-level analysis of multiple randomized trials (SEAL). J Clin Oncol. 2018;36:2593-602.

33. Yang Y, Cao JZ, Lan SM, Wu JX, Wu T, Zhu SY, et al. Association of improved locoregional control with prolonged survival in early-stage extranodal nasal-type natural killer/T-cell lymphoma. JAMA Oncol. 2017;3:83-91.

34. Liu X, Wu T, Zhu SY, Shi M, Su H, Wang Y, et al. Riskdependent conditional survival and failure hazard after radiotherapy for early-stage extranodal natural killer/T-cell lymphoma. JAMA Netw Open. 2019;2:e190194.
35. Yamaguchi M, Suzuki R, Kim SJ, Ko YH, Oguchi M, Asano N, et al. Early disease progression in patients with localized natural killer/T-cell lymphoma treated with concurrent chemoradiotherapy. Cancer Sci. 2018;109:2056-62.

36. Lim SH, Hong JY, Lim ST, Hong H, Arnoud J, Zhao W, et al. Beyond first-line non-anthracycline-based chemotherapy for extranodal NK/T-cell lymphoma: clinical outcome and current perspectives on salvage therapy for patients after first relapse and progression of disease. Ann Oncol. 2017;28:2199-205.

37. Qi SN, Yang Y, Zhang YJ, Huang HQ, Wang Y, He X, et al. Risk-based, response-adapted therapy for early-stage extranodal nasal-type NK/T-cell lymphoma in the modern chemotherapy era: a China Lymphoma Collaborative Group (CLCG) study. Am J Hematol. 2020;95:1047-56.

38. Chen B, Zhu SY, Shi M, Su H, Wang Y, He X, et al. Riskdependent curability of early-stage extranodal nasal-type NK/Tcell lymphoma in response to radiotherapy in elderly patients: a multicenter study from the China Lymphoma Collaborative Group (CLCG). Cancer Med. 2018;7:5952-61.

39. Casulo C, Byrtek M, Dawson KL, Zhou X, Farber CM, Flowers $\mathrm{CR}$, et al. Early relapse of follicular lymphoma after rituximab plus cyclophosphamide, doxorubicin, vincristine, and prednisone defines patients at high risk for death: an analysis from the National LymphoCare Study. J Clin Oncol. 2015;33:2516-22.

40. Zhu J, Yang Y, Tao J, Wang SL, Chen B, Dai JR, et al. Association of progression-free or event-free survival with overall survival in diffuse large B-cell lymphoma after immunochemotherapy: a systematic review. Leukemia. 2020. https://doi.org/10. 1038/s41375-020-0963-1.

41. Luminari S, Merli M, Rattotti S, Tarantino V, Marcheselli L, Cavallo F, et al. Early progression as a predictor of survival in marginal zone lymphomas: an analysis from the FIL-NF10 study. Blood. 2019;134:798-801.

\section{Affiliations}

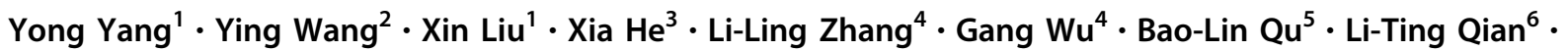

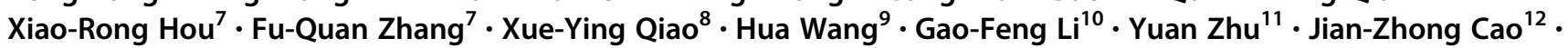 Jun-Xin $\mathrm{Wu}^{13} \cdot \mathrm{Tao}_{\mathrm{Wu}^{14}} \cdot \mathrm{Su}-\mathrm{Yu} \mathrm{Zhu}^{15} \cdot \mathrm{Mei} \mathrm{Shi}^{16} \cdot \mathrm{Li}-\mathrm{Ming} \mathrm{Xu} \mathbb{1}^{17} \cdot \mathrm{Hang} \mathrm{Su}^{18} \cdot \mathrm{Yu}-\mathrm{Qin}$ Song ${ }^{19} \cdot$ Jun $^{17} \mathrm{Zh}^{19}$. Yu-Jing Zhang ${ }^{20} \cdot$ Hui-Qiang Huang ${ }^{20} \cdot$ Chen Hu $\mathbb{D}^{21} \cdot$ Shu-Nan Qi $\oplus^{1} \cdot$ Ye-Xiong Li $\oplus^{1}$}

1 State Key Laboratory of Molecular Oncology and Department of Radiation Oncology, National Cancer Center/Cancer Hospital, Chinese Academy of Medical Sciences (CAMS) and Peking Union Medical College (PUMC), Beijing, China

2 Chongqing University Cancer Hospital \& Chongqing Cancer Hospital, Chongqing, China

3 Jiangsu Cancer Hospital \& Jiangsu Institute of Cancer Research, Nanjing, Jiangsu, China

4 Union Hospital, Tongji Medical College, Huazhong University of Science and Technology, Wuhan, Hubei, China

5 The General Hospital of Chinese People's Liberation Army, Beijing, China

6 The Affiliated Provincial Hospital of Anhui Medical University, Hefei, Anhui, China

7 Peking Union Medical College Hospital, Chinese Academy of Medical Sciences (CAMS) and Peking Union Medical College (PUMC), Beijing, China
8 The Fourth Hospital of Hebei Medical University, Shijiazhuang, China

9 Second Affiliated Hospital of Nanchang University, Nanchang, China

10 Beijing Hospital, National Geriatric Medical Center, Beijing, China

11 Cancer Hospital of the University of Chinese Academy of Sciences, Zhejiang Cancer Hospital, Hangzhou, Zhejiang, China

12 Shanxi Cancer Hospital and the Affiliated Cancer Hospital of Shanxi Medical University, Taiyuan, Shanxi, China

13 Fujian Provincial Cancer Hospital, Fuzhou, Fujian, China

14 Affiliated Hospital of Guizhou Medical University, Guizhou Cancer Hospital, Guiyang, Guizhou, China

15 Hunan Cancer Hospital and the Affiliated Cancer Hospital of Xiangya School of Medicine, Changsha, Hunan, China 
16 Xijing Hospital of Fourth Military Medical University, Xi'an, China

17 Tianjin Medical University Cancer Institute \& Hospital, Key Laboratory of Cancer Prevention and Therapy, National Clinical Research Center for Cancer, Tianjin, China

18 The Fifth Medical Center of PLA General Hospital, Beijing, China

19 Key Laboratory of Carcinogenesis and Translational Research (Ministry of Education), Peking University Cancer Hospital \& Institute, Beijing, China
20 Sun Yat-Sen University Cancer Center; State Key Laboratory of Oncology in South China; Collaborative Innovation Center for Cancer Medicine, Guangzhou, Guangdong, China

21 Division of Biostatistics and Bioinformatics, Sidney Kimmel Comprehensive Cancer Center, Johns Hopkins University School of Medicine, Baltimore, MD 21205-2013, USA 